\title{
Knochenzemente für die Behandlung osteoporotischer Frakturen
}

Ladina Hofmann-Fliri, Markus Windolf

\section{Warum Zement in der Frakturversorgung?}

Knochenzemente sind Biomaterialien, die im flüssigen Zustand injiziert werden können und im Körper aushärten, um hauptsächlich mechanische Aufgaben zu übernehmen. Ähnlich einem Dübel geht es dabei um eine Verbesserung der Kraftübertragung zwischen lasttragenden Komponenten, um einem mechanischen Versagen vorzubeugen. Solch eine invasive Prozedur birgt offensichtlich Risiken, deren Rechtfertigung im starken Anstieg von osteoporotischen Frakturen liegt, welcher in der Zukunft die Anpassung unserer Behandlungsstrategien erfordern wird.

Osteoporose ist eine systemische Skeletterkrankung, bei der eine Verringerung der Knochenmasse und eine Verschlechterung der Mikroarchitektur des Knochens festzustellen ist ( $\triangleright$ Abb. 1). Es finden abnormale Umstrukturierungen der trabekulären Knochenstruktur in Form einer Abnahme der Anzahl und Größe der Knochenbälkchen sowie eine Degression der Kortikalis statt [1]. Osteoporose ist die häufigste Skeletterkrankung und tritt mit zunehmender Wahrscheinlichkeit im höheren Lebensalter auf. Der Studie von Hadji et al. [2] zufolge litten im Jahr 2009 ca. 6,3 Millionen über 50-Jährige Deutsche an dieser Krankheit. Weltweit sind mehr als 200 Millionen Menschen betroffen. Etwa $80 \%$ der Betroffenen sind Frau-
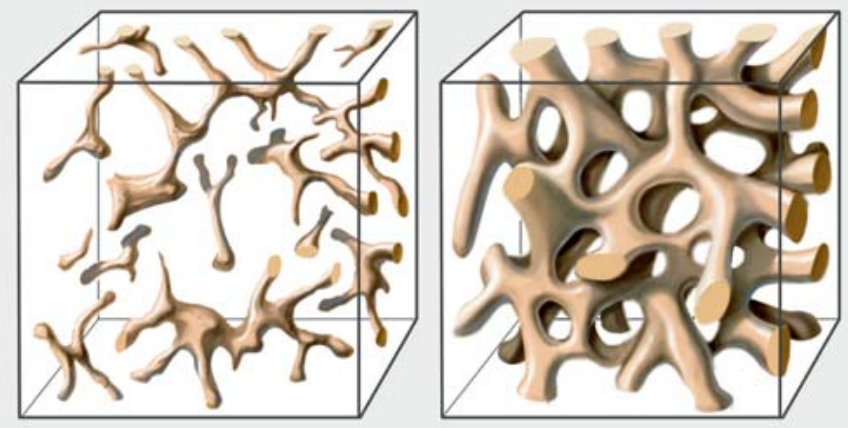

- Abb. 1 Dreidimensionale Mikroarchitektur der Spongiosa, links Osteoporose, rechts normaler Knochen. Quelle: Bartl R. Osteoporose. In Bartl R. Hrsg. Klinische Osteologie. 1. Aufl. Stuttgart: Thieme 2014 en. Damit erkrankt jede 4. Frau über 50 Jahre an Osteoporose. In Europa ergibt sich bei Frauen und Männern zwischen 50 und 79 Jahren eine Osteoporoseprävalenz von $12 \%$ [3]. Jede 2. an Osteoporose erkrankte Person erleidet im Laufe ihres Lebens eine darauf zurückzuführende Fraktur [4]. Aufgrund der steigenden Lebenserwartung der Bevölkerung ist zukünftig von einer höheren Prävalenz osteoporotischer Frakturen auszugehen [5]. Vor allem für ältere Personen kann eine Fraktur zu einer lebensbedrohlichen Situation führen, vor allem, wenn Mobilität und Unabhängigkeit stark beeinträchtigt werden [4].

\section{Merke}

Die Fixation solcher Frakturen mittels metallischer Implantate erreicht oft ihre Grenzen, was zu postoperativen Komplikationen und Revisionsoperationen führt.

Die Komplikationsrate bei proximalen Femurfrakturen, die mit einer dynamischen Hüftschraube versorgt sind, liegt bspw. bei $12 \%$ in der älteren Bevölkerung [6]. Am proximalen Humerus sind die Komplikationsraten höher [7-11]. Die Sterblichkeitsrate im 1. Jahr nach einer Refixation (dynamische Hüftschraube als primäre Behandlung) liegt bei 56\% [12].

Mechanisch gesehen führt ein verminderter Halt des Implantats in spongiösen Knochenregionen zum Versagen der Fixation. Wiederkehrende Belastungen durch physiologische Kräfte führen zur Ausbreitung von Mikrorissen und schlussendlich zum Versagen der lasttragenden Struktur. Eine Revisionsoperation wird unausweichlich.

Eine mögliche Lösung, um eine gute Verankerung sicherzustellen, ist die mechanische Verstärkung der geschwächten Knochenstruktur mit Knochenzement, die sog. Implantataugmentation [13]. Im Gegensatz zu einer systemischen Medikation mittels bspw. Bisphosphonaten, bietet Zement den großen Vorteil einer sofortigen und langzeitigen Wirkung.

\section{Zementarten}

Die gängigsten Knochenzemente sind Acrylate, wie z. B. Polymethylmethacrylate (PMMA), umgangssprachlich „Plexiglas“ genannt, die aus einer flüssigen und pulvrigen 
Komponente vor Gebrauch gemischt werden. Die chemische Reaktion verläuft exotherm, d. h. sie findet unter Freisetzen von Wärme statt. PMMA-Knochenzement wurde erstmals Mitte des letzten Jahrhunderts in der plastischen Chirurgie angewendet, um Lücken in Schädeln zu schließen. Aufgrund der guten körperlichen Verträglichkeit wurde das Einsatzgebiet daraufhin erweitert. So wurden z. B. Endoprothesen mit PMMA im Knochen fixiert und damit hohe Passgenauigkeit der Prothese und rasche Stabilität des Gelenks erzeugt $[14,15]$. Ermutigt durch die bis jetzt guten Erfahrungen, entsprang die Idee, das Konzept der Verstärkung mittels Knochenzement auch im Bereich der Frakturversorgung anzuwenden $[13,16]$. Die Nutzung von PMMA für die osteoporotische Frakturversorgung (Implantataugmentation) wird allerdings noch skeptisch betrachtet und ist dementsprechend noch nicht vollends in der klinischen Routine etabliert. Ein wichtiger Grund für die Zurückhaltung ist die Irreversibilität der Prozedur. Einmal injiziert verbleibt der Zement permanent im Körper. Da die stabilisierende Funktion des Zements nach einer Heilungszeit von 2-6 Monaten obsolet wird, stellt sich die Frage, ob dieser Nachteil gerechtfertigt ist. Die hohen Komplikationsraten und der weltweite Anstieg von osteoporotischen Patienten sprechen klar dafür.

Alternativen zu PMMA sind biologisch abbaubare Materialien wie Kalziumphosphatzemente (CaP-Zemente) $[17,18]$ oder andere in der Entwicklung befindliche Zusammensetzungen [19]. CaP-Zemente stellen eine spezielle Gruppe unter den Knochenersatzstoffen dar. Sie bestehen im Wesentlichen aus Kalziumphosphatverbindungen, die nach Anrühren mit einer wässrigen Lösung in ein- bzw. mehrstufigen Reaktionen bei Raumtemperatur unter Phasenumwandlung der Reaktanden zur Aushärtung des Zements führen. CaP-Zemente sind osteokonduktiv und werden durch Osteoklasten resorbiert und schließlich durch körpereigenen Knochen langsam ersetzt. Die Verwendung von CaP als biokompatibler Zementwerkstoff wurde erstmals 1982 von LeGeros et al. [20] vorgeschlagen. Im Jahr 1986 wurde dann der erste in situ abbindende CaP-Zement entwickelt [21], gefolgt von zahlreichen Untersuchungen verschiedener Zusammensetzungen auf ihre Eignung als Knochenersatzstoff [22]. In den 90er-Jahren wurde erstmals die Verwendung von CaP-Zement für die Implantataugmentation untersucht [23-25].

\section{Mechanische Eigenschaften}

Mechanische Komplikationen in der Alterstraumatologie sind fast ausschließlich auf Überbelastung des geschwächten Knochens - nicht des Implantats - zurückzuführen. Zement in der Implantataugmentation soll somit die trabekuläre Struktur um das Implantat verstärken [26]. Mechanische Eigenschaften von Zementen liegen

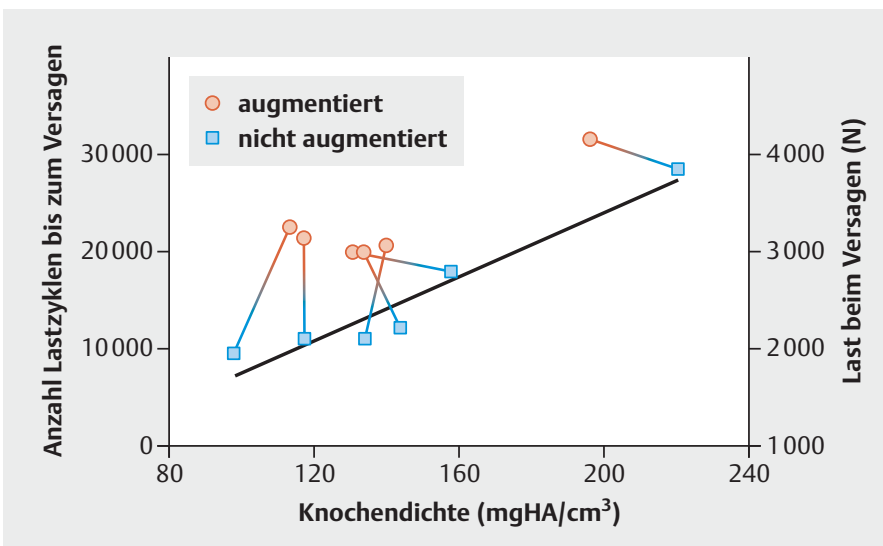

- Abb. 2 Korrelation der Anzahl Lastzyklen (bzw. Last) beim Versagen mit der Knochendichte unter experimenteller Wechselbelastung. Die Augmentation scheint den Effekt der schlechten Knochenqualität (Osteoporose) zu eliminieren (Sermon et al. [27], mit freundlicher Genehmigung von Wolters Kluwer Health, Inc.)

deutlich unterhalb von Metallen, aber oberhalb von Knochen.

\section{Merke}

Ein Zementmantel um das Implantat erlaubt daher eine „sanfte“ Übertragung physiologischer Kräfte von der knöchernen Struktur in das Implantat.

Der biomechanische Nutzen kann dadurch erklärt werden, dass die Belastung in der Knochenstruktur verringert wird, indem sich die lasttragende Fläche vergrößert $[26,27]$. Zusätzlich werden durch scharfe metallische Kanten verursachte Spannungsspitzen und Kerbwirkungen sowie abrupte Übergänge von Metall zu Knochen eliminiert [27]. Kleine Mengen PMMA-Zement (2-3 ml) im Femurkopf in Kombination mit dynamischer Hüftschraube (DHS, DePuySynthes GmbH) [26] oder Klinge (PFNA, DePuySynthes $\mathrm{GmbH}$ ) [27-30] zeigen im Experiment eine deutliche Verbesserung der Implantatverankerung im porotischen Knochen unter wiederkehrender Belastung.

\section{Merke}

Dabei fiel der Effekt der Augmentation umso deutlicher aus, je schlechter die Knochenqualität war

( A Abb. 2).

Resultate aus klinischen Studien bestätigen die experimentellen Erkenntnisse [31,32]. Das Prinzip lässt sich auf andere Anwendungen und anatomische Regionen, wie z. B. Schulter, Knie, Fuß und Becken übertragen [3339]. Die Resultate zeigen aber, dass der Nutzen stark von der individuellen Anwendung abhängig ist und die Augmentation immer im Gesamtkontext des individuellen Falles zu betrachten ist [40]. 
Acrylzemente besitzen ausgewogene mechanische Eigenschaften betreffend Steifigkeit, Bruch-/Dauerfestigkeit und Sprödbrüchigkeit und sind deshalb gut geeignet für lasttragende Anwendungen. Die Mechanik variiert allerdings je nach Belastungsrichtung. So sind PMMA-Zemente z. B. deutlich stabiler unter Druck- als unter Zugbelastungen.

Besonders die Scher- und Zugeigenschaften der CaP-Zemente sind deutlich geringer als die der PMMA-Zemente. Kalziumphosphate sind von Natur aus sprödbrüchig. Versuche, die Eigenschaften von CaP-Zementen zu verbessern, waren bisher nur mäßig erfolgreich [41,42] und führten oft zu Einbußen in der Biokompatibilität [43]. Außerdem wurden neue Zusammensetzungen meist nur in osteoporotischen Knochenmodellen [44] oder unter statischer Belastung bis zum Versagen untersucht $[18,25]$. Es existieren nur wenige Studien zu den Ermüdungseigenschaften von CaP-Zementen [19,45].

\section{Merke}

Es wird oft geraten, die derzeit verfügbaren CaPZemente nur an nicht oder wenig belasteten Stellen anzuwenden.

Im Rahmen einer Weiterentwicklung der CaP-Zemente haben jedoch verschiedene Autoren innovative Methoden vorgestellt, um kontrolliert neue komplexe Strukturen herzustellen, die deutlich verbesserte biomechanische Eigenschaften erreichen [46-48]. Diese und weitere Entwicklungen [49] deuten darauf hin, dass CaP-Zemente in Zukunft eine größere Rolle in der Implantataugmentation spielen könnten.

\section{Temperaturverhalten}

Acrylzemente härten unter Entstehung von Wärme aus. Der Temperaturanstieg kann zu Nekrosen des Knochens bzw. des angrenzenden Knorpels führen. Es ist daher wichtig, die Menge an eingespritztem Material klein zu halten. Verschiedene experimentelle Studien konnten zeigen, dass mit den erwähnten kleinen Mengen an PMMA keine kritischen Temperaturwerte überschritten werden [50-52] ( A Abb. 3).

Im Vergleich zu PMMA-Zementen setzt sich die von CaPZementen herrührende Reaktionswärme viel langsamer frei, was einen kleineren Temperaturanstieg im Zement bewirkt. Somit ist das Risiko von Temperaturnekrosen deutlich gemindert.

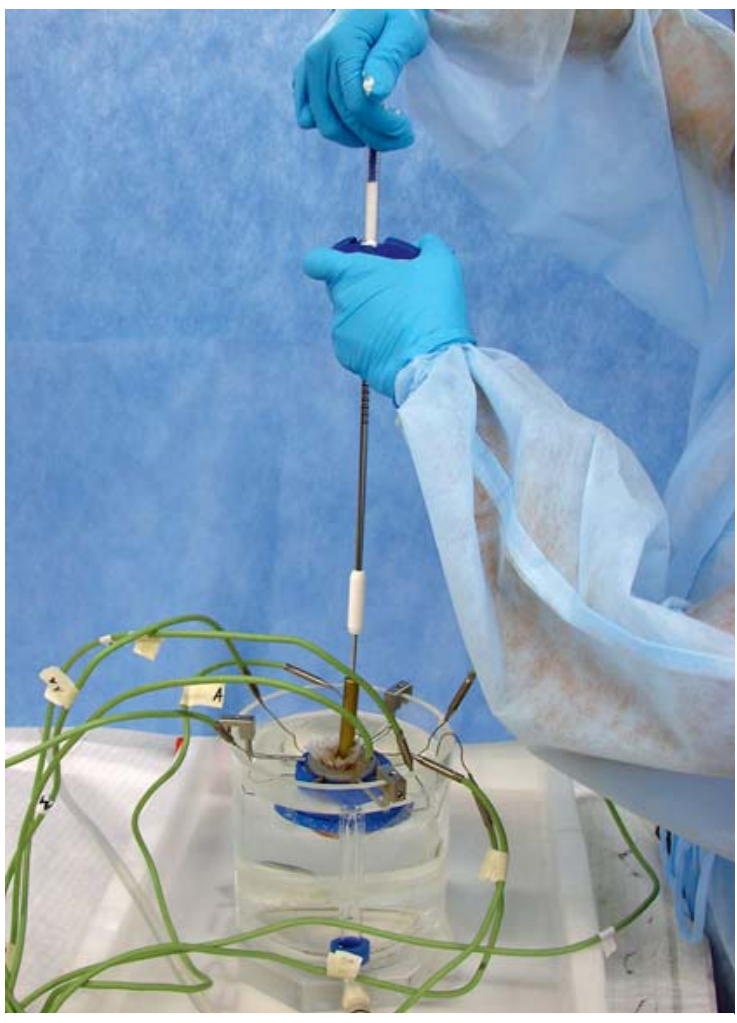

- Abb. 3 Messung der Temperaturen in einem menschlichen Femurkopf während der Injektion von 6 ml PMMAZement durch eine PFNA-Klinge (DePuy Synthes $\mathrm{GmbH}$ ). Um physiologische Bedingungen zu simulieren, liegen die Proben in einem $37-{ }^{\circ} \mathrm{C}$-Wasserbad. Die Temperatursensoren sind radial um die Probe angebracht und messen in verschiedenen Tiefen, um Temperaturwerte nah und fern der Zementwolke zu erfassen [52].

\section{Biokompatibilität}

Die hohe Körperverträglichkeit von Acrylaten wurde während des Zweiten Weltkriegs festgestellt, als Plexiglassplitter, z. B. von geborstenen Cockpitscheiben, bei verwundeten Soldaten umstandslos einheilten und sich verkapselten [53]. Dennoch stand die Frage im Raum, ob die Präsenz einer Zementwolke im knorpelnahen Bereich die Durchblutung und Versorgung des subchondralen Knochens und des darüber liegenden Knorpels beeinträchtigt.

\footnotetext{
Merke

In einer Schafsstudie konnte gezeigt werden, dass die aus biomechanischer Sicht vorteilhafte gelenknahe Platzierung einer Zementwolke kurz- und mittelfristig keinen negativen Einfluss auf den Knorpel zu haben scheint [54].
}

Langfristige klinische Erfahrungen müssen folgen. 


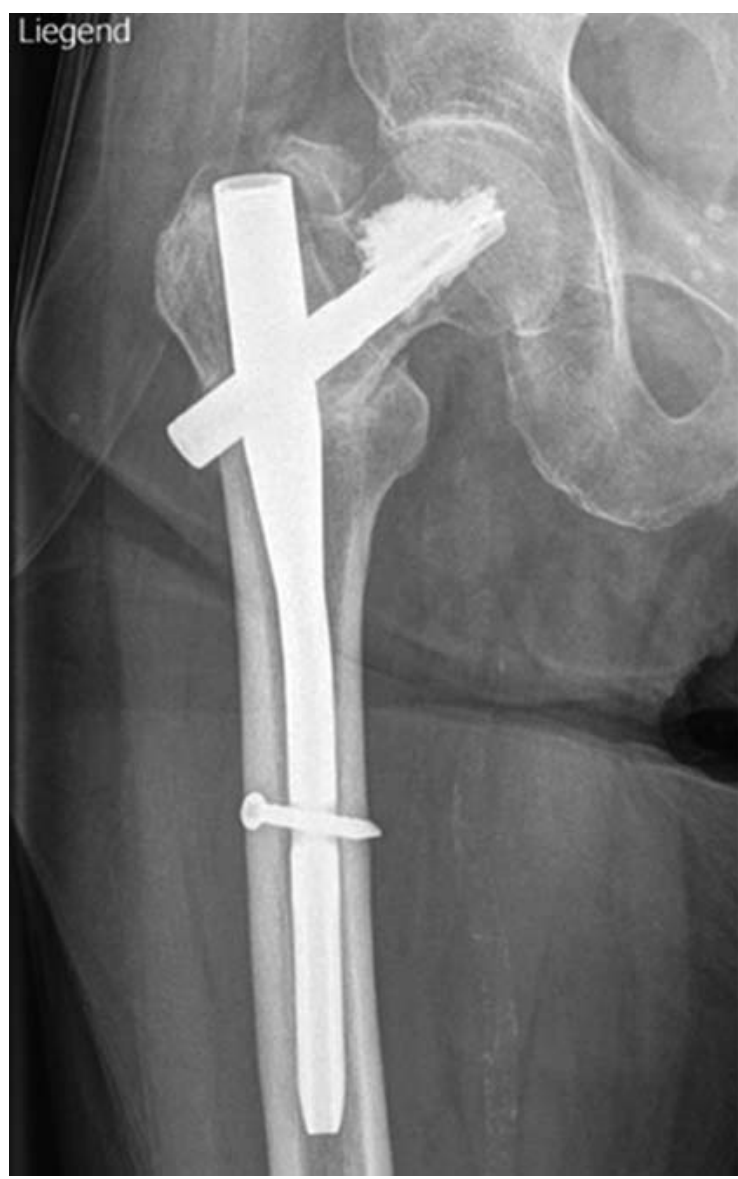

- Abb. 4 Augmentation bei einem proximalen Femurnagel. Quelle: Gosch M, Kammerlander C, Roth T et al. Alterstraumatologie - aktuelle Aspekte der interdisziplinären Betreuung von Patienten mit Fragilitätsfrakturen. DMW 2014; 139: 1207-1210

Bedenkt man, dass menschliches Knochengewebe zu $70 \%$ aus Kalziumphosphat besteht, ist offensichtlich, dass sich CaP-basierte Zemente aus biologischer Sicht ideal für die Verstärkung von Knochenstrukturen eignen. Die Eigenschaften von CaP-Zementen können durch eine große Zahl von Parametern beeinflusst werden. Anstrengungen, die Eigenschaften von CaP-Zementen durch Zusätze oder spezielle Formulierungen zu optimieren, können jedoch auch zu Einbußen in der Biokompatibilität führen [55-57].

Im Gegensatz zu PMMA-Zementen zeigen CaP-Zemente eine langsame Resorption des Werkstoffs, einhergehend mit dem Ersatz durch Knochenneubildung ohne Verlust an Volumen. Derzeit ist noch nicht klar, wie die CaP-Resorption kontrolliert werden kann [58], und die Frage bleibt, wie viel Knochen sich bei osteoporotischen Patienten nach der Resorption des Zements wieder bildet.

\section{Handhabung}

Vorbereitende Prozesse, wie das Anmischen von Zement und das Füllen von Spritzen, sollten einfach und zuverlässig zu bewerkstelligen sein. Die meisten Zemente erfüllen diese Anforderungen. Jedoch besteht besonders bei manchen CaP-Zementen noch Verbesserungspotenzial.

Um während des Einspritzens ein Austreten von Zement in kritische Regionen, wie z. B. im Gelenk- oder Frakturspalt, zu vermeiden, ist die Fließcharakteristik ein wichtiger Parameter $[59,60]$. Dabei spielt der Zusammenhang zwischen Viskosität und Ausbreitungsverhalten des Zements eine wichtige Rolle. Je höher die Viskosität, desto homogener verteilt sich der Zement im Knochen und desto geringer ist die Gefahr des Zementausflusses [59, 61]. Jedoch erfordert eine höhere Viskosität eine dementsprechend höhere Injekionskraft [62]. Bei der Formulierung des Zements gilt es nun, einen Kompromiss zwischen beiden Aspekten sowie der Mechanik zu schaffen [63].

Bei PMMA-Zementen sollte idealerweise vor Aushärtung eine mittlere bis hohe Viskosität für ein Zeitfenster von ungefähr 20 Minuten konstant aufrecht erhalten bleiben, um eine sichere Anwendung im OP zu gewährleisten [59]. Dieses Verhalten führt aber oft auch zu Einbußen in der Bruchfestigkeit, insbesondere unter Zugbelastung [65].

PMMA-Zemente lassen sich sehr gut injizieren, während CaP-Zemente eine Tendenz zur Phasentrennung haben. Deren Injizierbarkeit kann verbessert werden durch Verwendung eines geringeren Pulver-Flüssigkeits-Verhältnisses [66], durch Hinzufügen viskositätsregulierender Zusätze oder Verwendung bestimmter Zusätze, um die Aushärtung zu verzögern [67]. Diese Maßnahmen können aber wiederum andere Eigenschaften negativ beeinflussen [57].

Um den Knochenzement unter Röntgen sichtbar zu machen, ist ihm ein Kontrastmittel zugesetzt. Da PMMA-Zemente nicht resorbierbar sind, können alle röntgendichten, nicht oder schwer löslichen Pulver, wie Metallsalze (z. B. Bariumsulfat) verwendet werden. Bei den CaP-Zementen ist das Problem größer, da sie langsam resorbiert werden. Somit werden alle hinzugefügten Pulver mit der Zeit freigesetzt, was eine Biokompatibilitätsgefahr darstellen kann. Das Mengenverhältnis Kontrastmittel zu Zement bestimmt stark die Aushärtungscharakteristik und das Fließverhalten und ist damit eine wichtige Stellschraube zur Zementkomposition.

Um die Sicherheit der Prozedur zu erhöhen, werden meist kanülierte und perforierte Implantate für die Augmentation mit PMMA verwendet. Der Zement wird durch 
Kanülierung und radiale Perforationen in die Knochenstruktur gedrückt, nachdem das Implantat platziert wurde ( $\triangleright$ Abb. 4). Setzt man umgekehrt ein Implantat in eine vorher applizierte Zementwolke, besteht das Risiko der Zementaushärtung, falls sich aus verschiedenen Gründen die Implantation verzögert. Weiterhin ist es gewünscht, die Entscheidung für oder wider eine Augmentation erst nach Einbringen des Implantats zu treffen. Es soll hierbei erwähnt werden, dass die erforderlichen Injektionskräfte mit der Knochendichte stark steigen, sodass es schwer ist, PMMA-Zement in gesunden Knochen zu injizieren. Die Augmentation kann (und soll) demnach nicht als Korrekturprozedur für ungenaue Implantatplatzierung dienen. Außerdem besteht häufig der Irrglaube, dass sich über die Injektionskraft am Spritzenstempel der Zementaustrittsdruck steuern lässt, wenn Zement über eine Spritze manuell injiziert wird [68].

\section{Schlussfolgerungen}

Die Zementaugmentation von Implantaten besitzt ein großes Potenzial, um die Versorgung von osteoporotischen Knochenbrüchen zu verbessern, da sie für eine direkte mechanische Stabilisierung der Situation sorgt. Grundsätzlich gibt es momentan 2 Alternativen für Knochenzemente, Acrylate (PMMA) und Kalziumphosphate. Der Hauptunterschied liegt in der Resorbierbarkeit der Kalziumphosphatlösungen gegenüber dem permanent verbleibenden PMMA. Es muss dabei entschieden werden, ob dieser Vorteil im Zielkollektiv der älteren Patienten die derzeitigen mechanischen und Handhabungsvorteile von PMMA überwiegt.

\section{Interessenkonflikt}

Die Autoren geben an, dass keine Interessenkonflikte bestehen.

\section{Über die Autoren}

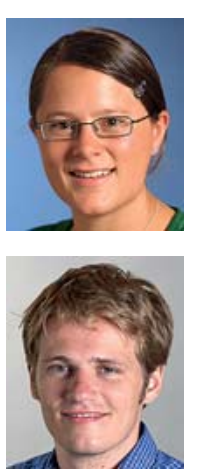

Ladina Hofmann-Fliri

Senior Project Leader, Concept Development, AO Forschungsinstitut Davos

\section{Dr. Markus Windolf}

Focus Area Leader, Concept Development, AO Forschungsinstitut Davos

\section{Korrespondenzadresse}

\author{
Ladina Hofmann-Fliri \\ Senior Project Leader \\ Concept Development \\ AO Forschungsinstitut Davos \\ Clavadelerstrasse 8 \\ 7270 Davos Platz \\ Schweiz \\ ladina.hofmann@aofoundation.org
}

\section{Literatur}

[1] Nordin BE. Osteoporosis With Particular Reference to the Menopause. In: Avioli LV, ed. The Osteoporotic Syndrome: Detection, Prevention and Treatment. New York: Grune and Stratton; 1983: 13-43

[2] Hadji P, Klein S, Gothe H et al. The epidemiology of osteoporosis - Bone Evaluation Study (BEST): an analysis of routine health insurance data. Dtsch Arztebl Int 2013; 110: 52-57

[3] Bartl R. Osteoporose. Prävention - Diagnostik - Therapie. Stuttgart: Thieme; 2010

[4] Court-Brown CM, Caesar B. Epidemiology of adult fractures: A review. Injury 2006; 37: 691-697

[5] Diepgen TL. [Demographic development of the population]. J Dtsch Dermatol Ges 2005; 3 (Suppl. 2): S36-S39

[6] Davis TR, Sher JL, Horsman A et al. Intertrochanteric femoral fractures. Mechanical failure after internal fixation. J Bone Joint Surg $\mathrm{Br} 1$ 1990; 72: 26-31

[7] Brunner F, Sommer C, Bahrs C et al. Open reduction and internal fixation of proximal humerus fractures using a proximal humeral locked plate: a prospective multicenter analysis. J Orthop Trauma 2009; 23: 163-172

[8] Roderer G, Erhardt J, Graf M et al. Clinical results for minimally invasive locked plating of proximal humerus fractures. J Orthop Trauma 2010; 24: 400-406

[9] Solberg BD, Moon CN, Franco DP et al. Locked plating of 3and 4-part proximal humerus fractures in older patients: the effect of initial fracture pattern on outcome. J Orthop Trauma 2009; 23: 113-119

[10] Sudkamp N, Bayer J, Hepp P et al. Open reduction and internal fixation of proximal humeral fractures with use of the locking proximal humerus plate. Results of a prospective, multicenter, observational study. J Bone Joint Surg Am 2009; 91: 1320-1328

[11] Voigt C, Woltmann A, Partenheimer A et al. [Management of complications after angularly stable locking proximal humerus plate fixation]. Chirurg 2007; 78: 40-46

[12] Palmer SJ, Parker MJ, Hollingworth W. The cost and implications of reoperation after surgery for fracture of the hip. J Bone Joint Surg Br 2000; 82: 864-866

[13] Bartucci EJ, Gonzalez MH, Cooperman DR et al. The effect of adjunctive methylmethacrylate on failures of fixation and function in patients with intertrochanteric fractures and osteoporosis. J Bone Joint Surg Am 1985; 67: 1094-1107

[14] Charnley J. Arthroplasty of the hip. A new operation. Lancet 1961; 1: 1129-1132

[15] Charnley J. A biomechanical analysis of the use of cement to anchor the femoral head prosthesis. J Bone Joint Surg Br 1965; 47: 354-363

[16] Augat P, Rapp S, Claes L. A modified hip screw incorporating injected cement for the fixation of osteoporotic trochanteric fractures. J Orthop Trauma 2002; 16: 311-316 
[17] Baroud G, Bohner M, Heini P et al. Injection biomechanics of bone cements used in vertebroplasty. Biomed Mater Eng 2004; 14: 487-504

[18] Elder S, Frankenburg E, Goulet J et al. Biomechanical evaluation of calcium phosphate cement-augmented fixation of unstable intertrochanteric fractures. J Orthop Trauma 2000; 14 : 386-393

[19] Ignatius AA, Augat P, Ohnmacht $M$ et al. A new bioresorbable polymer for screw augmentation in the osteosynthesis of osteoporotic cancellous bone: a biomechanical evaluation. J Biomed Mater Res 2001; 58: 254-260

[20] LeGeros RZ, Chohayeb A, Schulmann A. Apatitic calcium phosphates: Possible restorative materials. J Dent Res 1982; 61 (Spec Issue): Abstr. No. 1482, 343

[21] Brown WE, Chow LC. A New Calcium Phosphate, Water-Setting Cement. In: Brown PW, ed. Cements Research Progress 1986. Westerville, OH: American Ceramic Society; 1987: 352-379

[22] Chow LC, Markovic M, Takagi S. Calcium Phosphate Cements. In: Struble LJ, ed. Cements Research Progress 1997. Westerville, OH: American Ceramic Society; 1998: 215-238

[23] Bohner M, Lemaître J, Cordey J et al. Potential use of biodegradable bone cement in bone surgery: holding strength of screws in reinforced osteoporotic bone. Orthopadic Trans 1992; 16: 401-402

[24] Goodman SB, Bauer TW, Carter D et al. Norian SRS cement augmentation in hip fracture treatment. Laboratory and initial clinical results. Clin Orthop Relat Res 1998; 348: 42-50

[25] Moore DC, Frankenburg EP, Goulet JA et al. Hip screw augmentation with an in situ-setting calcium phosphate cement: an in vitro biomechanical analysis. J Orthop Trauma 1997; 11: 577583

[26] von der Linden P, Gisep A, Boner V et al. Biomechanical evaluation of a new augmentation method for enhanced screw fixation in osteoporotic proximal femoral fractures. J Orthop Res 2006; 24: 2230-2237

[27] Sermon A, Boner V, Boger A et al. Potential of polymethylmethacrylate cement-augmented helical proximal femoral nail antirotation blades to improve implant stability - a biomechanical investigation in human cadaveric femoral heads. J Trauma Acute Care Surg 2012; 72: E54-E59

[28] Erhart S, Schmoelz W, Blauth M et al. Biomechanical effect of bone cement augmentation on rotational stability and pullout strength of the Proximal Femur Nail Antirotation ${ }^{\mathrm{TM}}$. Injury 2011; 42: 1322-1327

[29] Fensky F, Nüchtern JV, Kolb JP et al. Cement augmentation of the proximal femoral nail antirotation for the treatment of osteoporotic pertrochanteric fractures - a biomechanical cadaver study. Injury 2013; 44: 802-807

[30] Sermon A, Boner V, Schwieger K et al. Biomechanical evaluation of bone-cement augmented Proximal Femoral Nail Antirotation blades in a polyurethane foam model with low density. Clin Biomech (Bristol, Avon) 2012; 27: 71-76

[31] Kammerlander C, Doshi H, Gebhard F et al. Long-term results of the augmented PFNA: a prospective multicenter trial. Arch Orthop Trauma Surg 2014; 134: 343-349

[32] Kammerlander C, Gebhard F, Meier C et al. Standardised cement augmentation of the PFNA using a perforated blade: A new technique and preliminary clinical results. A prospective multicentre trial. Injury 2011; 42: 1484-1490

[33] Goetzen M, Nicolino T, Hofmann-Fliri L et al. Metaphyseal screw augmentation of the LISS-PLT plate with polymethylmethacrylate improves angular stability in osteoporotic proximal third tibial fractures: a biomechanical study in human cadaveric tibiae. J Orthop Trauma 2014; 28: 294-299
[34] Grüneweller N, Raschke M], Zderic I et al. Biomechanical comparison of augmented versus non-augmented sacroiliac screws in a new hemi-pelvis test model. J Orthop Res 2016; DOI: 10.1002/jor.23401

[35] Kathrein S, Kralinger F, Blauth M et al. Biomechanical comparison of an angular stable plate with augmented and non-augmented screws in a newly developed shoulder test bench. Clin Biomech (Bristol, Avon) 2013; 28: 273-277

[36] Klos K, Wahnert D, Gueorguiev B et al. Development of a technique for cement augmentation of nailed tibiotalocalcaneal arthrodesis constructs. Clin Biomech (Bristol, Avon) 2010; 25: 576-581

[37] Rausch S, Klos K, Wolf U et al. A biomechanical comparison of fixed angle locking compression plate osteosynthesis and cement augmented screw osteosynthesis in the management of intra articular calcaneal fractures. Int Orthop 2014; 38 : 1705-1710

[38] Roderer G, Scola A, Schmolz W et al. Biomechanical in vitro assessment of screw augmentation in locked plating of proximal humerus fractures. Injury 2013; 44: 1327-1332

[39] Wahnert D, Hofmann-Fliri L, Richards RG et al. Implant augmentation: adding bone cement to improve the treatment of osteoporotic distal femur fractures: a biomechanical study using human cadaver bones. Medicine (Baltimore) 2014; 93: e166

[40] Hofmann-Fliri L, Nicolino TI, Barla J et al. Cement augmentation of implants-no general cure in osteoporotic fracture treatment. A biomechanical study on non-displaced femoral neck fractures. J Orthop Res 2016; 34: 314-319

[41] Juvonen T, Koistinen A, Kroger $\mathrm{H}$ et al. Enhanced bone screw fixation with biodegradable bone cement in osteoporotic bone model. J Appl Biomater Funct Mater 2012; 10: 113-118

[42] Wang M, Joseph R, Bonfield W. Hydroxyapatite-polyethylene composites for bone substitution: effects of ceramic particle size and morphology. Biomaterials 1998; 19: 2357-2366

[43] Ignatius AA, Betz O, Augat $P$ et al. In vivo investigations on composites made of resorbable ceramics and poly(lactide) used as bone graft substitutes. J Biomed Mater Res 2001; 58: 701-709

[44] Collinge C, Merk B, Lautenschlager EP. Mechanical evaluation of fracture fixation augmented with tricalcium phosphate bone cement in a porous osteoporotic cancellous bone model. J Orthop Trauma 2007; 21: 124-128

[45] Gisep A, Kugler S, Wahl D et al. Mechanical characterisation of a bone defect model filled with ceramic cements. J Mater Sci Mater Med 2004; 15: 1065-1071

[46] Bouville F, Maire E, Meille S et al. Strong, tough and stiff bioinspired ceramics from brittle constituents. Nat Mater 2014; 13: $508-514$

[47] Juvonen T, Nuutinen JP, Koistinen AP et al. Biomechanical evaluation of bone screw fixation with a novel bone cement. Biomed Eng Online 2015; 14: 74

[48] Munch E, Launey ME, Alsem DH et al. Tough, bio-inspired hybrid materials. Science 2008; 322: 1516-1520

[49] Habraken W, Habibovic P, Epple M et al. Calcium phosphates in biomedical applications: materials for the future? Materials Today 2016; 19: 69-87

[50] Blazejak M, Hofmann-Fliri L, Buchler L et al. In vitro temperature evaluation during cement augmentation of proximal humerus plate screw tips. Injury 2013; 44: 1321-1326 
[51] Boner V, Kuhn P, Mendel T et al. Temperature evaluation during PMMA screw augmentation in osteoporotic bone - an in vitro study about the risk of thermal necrosis in human femoral heads. J Biomed Mater Res B Appl Biomater 2009; 90: 842848

[52] Fliri L, Lenz M, Boger A et al. Ex vivo evaluation of the polymerization temperatures during cement augmentation of proximal femoral nail antirotation blades. J Trauma Acute Care Surg 2012; 72: 1098-1101

[53] Epple M. Biomaterialien und Biomineralisation. Eine Einführung für Naturwissenschafter, Mediziner und Ingenieure. Wiesbaden: B. G. Teubner; 2003

[54] Goetzen M, Hofmann-Fliri L, Arens D et al. Does metaphyseal cement augmentation in fracture management influence the adjacent subchondral bone and joint cartilage?: an in vivo study in sheep stifle joints. Medicine (Baltimore) 2015; 94: e414

[55] Kilian O, Wenisch S, Karnati S et al. Observations on the microvasculature of bone defects filled with biodegradable nanoparticulate hydroxyapatite. Biomaterials 2008; 29: 3429-3437

[56] Malard O, Bouler JM, Guicheux J et al. Influence of biphasic calcium phosphate granulometry on bone ingrowth, ceramic resorption, and inflammatory reactions: preliminary in vitro and in vivo study. J Biomed Mater Res 1999; 46: 103-111

[57] Miyamoto $\mathrm{Y}$, Ishikawa K, Takechi M et al. Histological and compositional evaluations of three types of calcium phosphate cements when implanted in subcutaneous tissue immediately after mixing. J Biomed Mater Res 1999; 48: 36-42

[58] Sheikh Z, Abdallah M-N, Hanafi AA et al. Mechanisms of in vivo degradation and resorption of calcium phosphate based biomaterials. Materials 2015; 8: 7913-7925

[59] Boger A, Wheeler K. A medium viscous acrylic cement enhances uniformity of cement filling and reduces leakage in cacellous bone augmentation. ISRN Mater Sci 2011; 2011 (780510)
[60] Boger A, Wheeler KD, Schenk B et al. Clinical investigations of polymethylmethacrylate cement viscosity during vertebroplasty and related in vitro measurements. Eur Spine J 2009; 18: $1272-1278$

[61] Bohner M, Gasser B, Baroud G et al. Theoretical and experimental model to describe the injection of a polymethylmethacrylate cement into a porous structure. Biomaterials 2003; 24: $2721-2730$

[62] Gisep A, Boger A. Injection biomechanics of in vitro simulated vertebroplasty - correlation of injection force and cement viscosity. Biomed Mater Eng 2009; 19: 415-420

[63] Deusser S, Sattig C, Boger A. Rheological and curing behavior of a newly developed, medium viscous acrylic bone cement. ISRN Mater Sci 2011; 2011 (571728)

[64] Zderic I, Steinmetz P, Benneker LM et al. Bone cement allocation analysis in artificial cancellous bone structures. J Orthop Translation 2017; 8: 40-48

[65] Boger A, Wheeler K, Montali A et al. NMP-modified PMMA bone cement with adapted mechanical and hardening properties for the use in cancellous bone augmentation. J Biomed Mater Res B Appl Biomater 2009; 90: 760-766

[66] Bohner M, Baroud G. Injectability of calcium phosphate pastes. Biomaterials 2005; 26: 1553-1563

[67] Zhang J, Liu W, Schnitzler V et al. Calcium phosphate cements for bone substitution: chemistry, handling and mechanical properties. Acta Biomater 2014; 10: 1035-1049

[68] Blankstein M, Widmer D, Gotzen M et al. Assessment of intraosseous femoral head pressures during cement augmentation of the perforated proximal femur nail antirotation blade. J Orthop Trauma 2014; 28: 398-402

\section{Bibliografie}

DOI http://dx.doi.org/10.1055/s-0043-102955 OP-JOURNAL 2017; 33: 56-62 @ Georg Thieme Verlag KG Stuttgart · New York ISSN 0178-1715 